\title{
Fingering Instability of Dislocations and Related Line Defects
}

\author{
Ming Li*†, Brian B. Smith*, and Robin L. B. Selinger* \\ ${ }^{*}$ Physics Department, Catholic University of America, Washington, DC 20064 \\ $\dagger$ Center for Nonlinear Science, Los Alamos National Laboratory, Los Alamos, New Mexico 87545
}

(October 9, 2018)

\begin{abstract}
We identify a fundamental morphological instability of mobile dislocations in crystals and related line defects. A positive gradient in the local driving force along the direction of defect motion destabilizes long-wavelength vibrational modes, producing a "fingering" pattern. The minimum unstable wavelength scales as the inverse square root of the force gradient. We demonstrate the instability's onset in simulations of a screw dislocation in $\mathrm{Al}$ (via molecular dynamics) and of a vortex in a 3-d XY "rotator" model.
\end{abstract}

PACS numbers: 61.72.Lk, 62.20.Fe, 75.10.Hk, 47.32.Cc

Modeling the motion of dislocations in crystals is mathematically challenging due to their long-range interaction through the elastic strain field 1 , and computational approaches include atomistic molecular dynamics2 3 and mesoscale models 1 . In some contexts the complexity of the problem can be reduced by approximating the motion of an individual dislocation as that of a damped elastic stringe, and such models can be used to describe the motion of other types of line defects as well, e.g. magnetic flux lines in type-II superconductors. Recent research using this approach has focused on locked vs. running behaviord of a line defect in a periodic potential due to the underlying crystal lattice. A morphological instability associated with a periodic driving force at a frequency resonant with the periodic potential has also been studiedls.

In the present Letter we identify a fingering instability in the glide of a mobile line defect under a spatially varying driving force that increases along the direction of motion. This work was motivated by unpublished molecular dynamics studies of screw dislocation pair annihilation in pure Cu performed by Swaminarayan and LeSare. The simulations appeared to show that initially straight parallel screw dislocations of opposite Burgers vector spontaneously developed a way profile prior to pair annihilation, something like the well known Crow instability of parallel vortices in fluid dynamics 10 . We were motivated by this result to consider whether the instability is driven by the attractive force gradient between the dislocations, and indeed whether a single dislocation could be unstable under a positive gradient in the driving stress. Since such stress gradients arise from common sources-e.g. external loading or dislocation interactions with a free surface or other microstructural features-such an instability might be active during metal deformation.

Consider the stability of an initially straight dislocation under an attractive force gradient, approximating its response as that of a damped elastic string. (The approximation is useful only to model the onset of any instability, because once the defect line deviates strongly from its initial state then nonlocal interactions can no longer be neglected.) The string's displacement obeys the familiar wave equation with linear damping and a spatially varying driving force:

$$
\frac{\partial^{2} \Psi}{\partial t^{2}}=c^{2} \frac{\partial^{2} \Psi}{\partial z^{2}}-\frac{\gamma}{m} \frac{\partial \Psi}{\partial t}+\frac{F(z, t)}{m} .
$$

Here $\Psi(z, t)$ is the displacement of the string in the $x$ direction, $c$ is the wave speed of an oscillation on the string, $\gamma$ is a linear damping coefficient, $m$ is the mass per unit length, and $F(z, t)$ is the driving force per unit length along the string. We take periodic boundary conditions with $z$ in the interval $(0, L)$. For our present purposes we do not include an underlying periodic potential; this is appropriate for modeling dislocation motion in FCC metals where the Peierls stress is small and unpinned dislocations are mobile even at low temperature.

We consider a driving force with a value that increases linearly in the direction the string is moving, as if the string were moving down a hill that gets continuously steeper. The force on the string at any point along its length may then be written:

$$
F(z, t)=F_{0}+F_{1} \Psi(z, t) .
$$

Any portion of the string that gets slightly ahead of the rest feels a stronger driving force. More generally Eqn. (2) represents the first two terms in an expansion, so that $F_{1}$ is defined as the local gradient of a spatially dependent driving force. If $F_{1}>0$ the force gradient is destabilizing and will cause a sinusoidal perturbation of sufficiently long wavelength to grow rather than die away, while small wavelength perturbations are suppressed by line tension, as shown below. 
To linearize the equation we switch to a (non-inertial) moving reference frame. Consider perturbations $\tilde{\Psi}(z, t)$ from a stable uniform running solution $\Psi_{0}(t)$,

$$
\Psi(z, t)=\Psi_{0}(t)+\tilde{\Psi}(z, t)
$$

where

$$
\frac{\partial^{2} \Psi_{0}}{\partial t^{2}}=-\frac{\gamma}{m} \frac{\partial \Psi_{0}}{\partial t}+\frac{F_{0}}{m}+\frac{F_{1}}{m} \Psi_{0}
$$

This procedure gives rise to the linearized equation of motion for the perturbation:

$$
\frac{\partial^{2} \tilde{\Psi}}{\partial t^{2}}=c^{2} \frac{\partial^{2} \tilde{\Psi}}{\partial z^{2}}-\frac{\gamma}{m} \frac{\partial \tilde{\Psi}}{\partial t}+\frac{F_{1}}{m} \tilde{\Psi}
$$

Taking a solution of the form $\tilde{\Psi}=A \mathrm{e}^{i(k z-\omega t)}$, we find the dispersion relation

$$
\omega(k)=-\frac{i \gamma}{2 m} \pm \frac{1}{2} \sqrt{\frac{-\gamma^{2}}{m^{2}}-4\left(\frac{F_{1}}{m}-c^{2} k^{2}\right)} .
$$

We identify three regions of $k$.

(1) Underdamped region: short wavelength $\left(k>k_{d}\right.$, or $\left.\lambda<\lambda_{d}\right)$ perturbations are underdamped, with

$$
k_{d}=\sqrt{\frac{\gamma^{2}}{4 c^{2} m^{2}}+\frac{F_{1}}{m c^{2}}} .
$$

We identify a characteristic length $\lambda_{d}=2 \pi / k_{d}$ as the wavelength associated with critical damping; modes with smaller wavelength are underdamped.

(2) Overdamped region: for intermediate wavelength $\left(k^{*}<k<k_{d}\right.$, or $\left.\lambda_{d}<\lambda<\lambda^{*}\right)$ perturbations are overdamped. The cut-off value $k^{*}$ is

$$
k^{*}=\sqrt{\frac{F_{1}}{m c^{2}}} .
$$

We identify the characteristic length $\lambda^{*}$

$$
\lambda^{*}=\frac{2 \pi}{k^{*}}=2 \pi \sqrt{\frac{m c^{2}}{F_{1}}} .
$$

Note that $\lambda^{*}$ decreases as the stress gradient $F_{1}$ increases, and that it is independent of the damping constant $\gamma$.

(3) Unstable region: long wavelength modes $\left(k<k^{*}\right.$, or $\left.\lambda>\lambda^{*}\right)$ are unstable, and will grow, leading to the formation of a "fingering" pattern. The fastest growing mode (which has largest positive Im $\omega$ ) is always that with the largest unstable wavelength.

To evaluate these characteristic lengths for a real material, we consider the case of a mobile screw dislocation in pure Al. The damping coefficient $\gamma$ has been estimated at about $10^{-4} \mathrm{~Pa}$-sed1, but in molecular dynamics simulation at temperatures close to zero we have found it to be about an order of magnitude smaller 2 . The wave speed is $c=29 \AA /$ psec and the effective mass depends logarithmically on system size but is in the range $g$ f $5 \mathrm{amu} / \AA \mathrm{for}$ a system of about $300 \times 300 \AA^{2}$ in cross section, again as found in a molecular dynamics simulation 12 . In the absence of a stress gradient, the wavelength associated with critical damping at low temperature is $\lambda_{d}=0.2$ to $0.3 \mu \mathrm{m}$; at higher temperatures where the damping is stronger, $\lambda_{d}$ may be as small as a few hundred $\AA$. The minimum unstable wavelength $\lambda^{*}$ depends on the local force gradient. For alumimum we estimate the energy/length of a screw dislocation as roughly $1 \mathrm{eV} / \AA$, and the driving force is $\sigma \cdot b$, where $\sigma$ is the driving stress and $b$ is the Burgers vector $=2.86 \AA$. Then the characteristic unstable wavelength can be estimated roughly as $\lambda^{*}=5 \times 10^{-4} \mathrm{~m}(\sigma / l)^{-1 / 2}$ where the stress gradient $\sigma / l$ is in units of $\mathrm{GPa} / \mathrm{m}$. Thus for a screw dislocation in $\mathrm{Al}$, we estimate that a stress gradient of $1 \mathrm{GPa} / \mathrm{cm}$ gives a minimum unstable wavelength of order $\lambda^{*}=50 \mu \mathrm{m}$, and a stress gradient of $100 \mathrm{GPa} / \mathrm{cm}$ gives $\lambda^{*}=5 \mu \mathrm{m}$.

If $\lambda^{*}<L$, where $L$ is the length of the defect line, then all vibrational modes with wavelength $\lambda>\lambda^{*}$ will be unstable. While the lowest unstable mode grows the fastest, in the early stages of growth all unstable modes are visible and give rise to a disordered fingering structure. If we follow the evolution of the linearized equation of motion in Eqn. (5) beyond the early stages of growth, we observe a coarsening process in which neighboring peaks in the 
structure merge until only a single sinusoid profile remains. In Fig. 1 we show the results of a numerical integration of Eqn. (5), showing the type of patterns that arise, where the initial condition was a straight line with a small amplitude random perturbation.

For dislocations in metals, the linearized equation is only valid to describe the very earliest stage of motion, so we would not necessarily expect to see fingering behavior like that shown in Fig. 1. However the initial growth of the instability should be described by the analysis above. To test this hypothesis, we performed a molecular dynamics simulation of the motion of a screw dislocation in a single crystal of aluminum using an Embedded Atom Method potential provided by Diana Farkas et al 1 . To see the instability in an atomic scale simulation is not an easy task, because to get a minimum unstable wavelength as small as $500 \AA$ in a bulk solid, we would need an enormous stress gradient in the range of $10^{8} \mathrm{GPa} / \mathrm{m}$. Luckily, there is a much easier way: we make use of the force gradient produced by the attraction between a dislocation line and a nearby free surface.

The force/length on a screw dislocation at a distance $R$ from a parallel free surface scales as $1 / R$, so the gradient in the driving force diverges as $1 / R^{2}$. When the dislocation is far enough from the surface, the local force gradient is small, the minimum unstable wavelength is longer than the dislocation line, and the dislocation is stable. At a critical distance from the wall $R^{*}$ (which depends on dislocation length $L$ ) the dislocation's first vibrational mode becomes unstable and fingers toward the free surface. As the dislocation continues to move toward the free surface, the force gradient gets stronger and higher order vibrational modes go unstable one by one in a cascade of instabilities.

Our simulated system has periodic boundary conditions only along the z-axis and thus has four free surfaces; the dislocation is initially parallel to the $\mathrm{z}$-axis which lies in the [110] direction. The system size is $45 \times 45 \times 183=370,575$ atoms, or $111 \times 105 \times 524 \AA^{3}$, and the temperature is close to $0^{\circ} \mathrm{K}$. The dislocation is placed a distance of $26.5 \AA$ from one free surface and is given a very slight sinusoidal perturbation with wavelength $\lambda=L=524 \AA$. We estimate the minimum unstable wavelength as:

$$
\lambda^{*}=2 \pi\left[\frac{\left(\log \left(\frac{2 R}{a_{o}}\right)+\log \left(\frac{2 D-2 R}{a_{o}}\right)-\log \left(\frac{2 D}{a_{o}}\right)\right)}{R^{-2}+(D-R)^{-2}}\right]^{1 / 2}
$$

where the numerator is the energy/length of the dislocation $\left(m c^{2}\right)$ and the denominator is the gradient of the driving force/length 1 , using image terms from free surfaces ahead and behind, and canceling the energy prefactor. Here $D=111 \AA$ is the length of the crystal in the direction of dislocation motion, and $a_{o}=b$ is the dislocation core size. For a dislocation at position $R=26.5 \AA$, Eqn. (10) predicts $\lambda^{*}=258 \AA$. Since $\lambda^{*}$ is about half the size of the defect length $L=524 \AA$, we expect both the first and second vibrational modes to be unstable and grow.

The dislocation is shown in Fig. 2, where we display only atoms whose local potential energy differs from that of the bulk. The initial state is shown in gray, the later state in black. The dislocation splits into two partial dislocations separated by a ribbon of stacking fault. In each of the partials, the initial sinusoidal perturbation grows along with the second vibrational mode, and each of the partials fingers toward the wall. The apparent minimum unstable wavelength is roughly $\lambda^{*}=250 \AA$, in agreement with our prediction. We have also run a simulation of the same set-up performed in a crystal of height $150 \AA$; in this case the initial perturbation does not grow, as its wavelength $\lambda=L<\lambda^{*}$, and the instability is suppressed by finite size.

Our simulation of a dislocation moving toward a free surface is closely related to Swaminarayan's 9 study of dislocation pair annihilation. In our case, the dislocation is attracted to an "image" dislocation symmetric position across the free surface. In order to see the longer time evolution of the instability, we need a larger system , so we have performed simulations of vortex motion in a three-dimensional XY "rotator" model of a simple magnet 14 . This system is very efficient computationally, and we have run simulations of up to $4 \times 10^{6}$ spins on a DEC Alpha workstation. In this model, each spin on a cubic lattice has a moment of inertia $I=1$ and one rotational degree of freedom with angular position $\theta_{i}$ and angular velocity $\omega_{i}$. The spins have nearest-neighbor interactions with an XY Hamiltonian, an applied magnetic field $B$, plus a rotational kinetic energy term:

$$
H=-\sum_{<i, j>} \cos \left(\theta_{i}-\theta_{j}\right)+\sum_{i}-B \cos \left(\theta_{i}\right)+\frac{1}{2} I \omega_{i}^{2} .
$$

We derive equations of motion from Eqn. (11) under constant energy. A straight vortex line has properties analogous to those of a screw dislocation in a crystal, particularly the effective mass/length, mobility behavior, Peierls barrier, and vortex-vortex interactions 12 .

We use periodic boundary conditions along the z direction only with four free surfaces, and size $100 \times 100 \times 400$ spins. In the initial state a vortex line lies parallel to the $\mathrm{z}$ axis at an initial temperature $k_{B} T=0.25$, in the ordered phase. Since the simulation is run at constant energy, $k_{B} T$ fluctuates. We apply a small exteral field $\mathrm{B}=-0.003$ which drives the vortex in the $+\mathrm{x}$ direction. 
In Fig. 3 we show the time evolution of the vortex, starting at $\mathrm{x}=40$ and moving toward the free surface at $\mathrm{x}=100$. Using Eqn.(10) with $a_{o}=1$ and $D=100$, we predict that the first vibrational mode should become unstable when the vortex reaches a distance $R=38$ lattice sites from the free surface. Since this system is at finite temperature, the vortex line roughens initially due to thermal fluctuations (like those studied by Chzran and Daw 15 in dislocations.) The first vibrational mode starts to grow at about the predicted location, around $\mathrm{x}=60$ ( $R=40$ units from the surface.) Around $x=65$, the second mode becomes prominent (with two "peaks" visible) and grows until the vortex annihilates at the free surface. The second mode appears earlier than predicted by Eqn. (10); perhaps nonlinear effects play an important role. In future work we plan to simulate a larger system in which more steps in the cascade of instabilities may be seen.

The physical signifigance of this instability is an open question. Most important, we have learned how finite size effects play a major role in simulations of line defects in general; and that the largest characteristic length is defined by the smallest force gradient present. Beyond that we speculate that the characteristic length scale $\lambda^{*}$ might play a role in the formation of dislocation patterns during work hardening. The instability could also control pair annihilation of threading dislocations in thin films, defining an effective "kill radius" that depends on film thickness 12 . Confirming any of these mechanisms via experiment may be difficult. The motion of an individual dislocation in a thin metal sample can be observed via TEM, but it is not clear whether the necessary time resolution and length scales are technically feasible. It would be easier to observe the motion of a defect line in a liquid crystal driven by a gradient in an applied field, since defect motion can be viewed with an optical microscope and polarizers.

A closely related instability has previously been identified by Z. Suo 16 who noted that a dislocation moving via climb can be unstable in the presence of a gradient in the density of vacancies. However since climb is a diffusive process, the dynamics of that instability mechanism occur on a far slower time scale where inertial effects are not important. However both mechanisms should play an important role in dislocation pattern formation in metals.

We thank Lyle Levine, Robb Thompson, and Shujia Zhou for helpful discussions. This work was supported by NSF Grant No. DMR-59702234.

${ }^{1}$ For a general introduction to dislocations and their interactions, see J. P. Hirth and J. Lothe, Theory of Dislocations (Krieger Publishing, Florida, 1992).

${ }^{2}$ S. J. Zhou, D. M. Beazley, P. S. Lomdahl, and B. L.Holian, Phys. Rev. Lett. 78, 479 (1997); S. J. Zhou, * D. L. Preston, P. S. Lomdahl, D. M. Beazley, Science 279, 1525 (1998).

${ }^{3}$ V. Bulatov, F. Abraham, L. Kubin, B. Devincre, S. Yip, Nature 391, 669 (1998).

${ }^{4}$ L. P. Kubin et al., Solid State Phenom. 23, 455 (1992).

${ }^{5}$ K. W. Schwarz, Phys. Rev. Lett. 78, 4785 (1997); K. W. Schwarz and F. K. LeGoues, Phys. Rev. Lett. 79, 1877 (1997).

${ }^{6}$ A. Granato and K. Lucke, J. Appl. Phys. 27, 583 (1956); J. Appl. Phys. 27, 789 (1956).

${ }^{7}$ C. Cattuto and F. Marchesoni, Phys. Rev. Lett. 79, 5070 (1997).

${ }^{8}$ E. B. Kolomeisky, T. Curcic,and J. P. Straley, Phys. Rev. Lett. 75, 1775 (1995).

${ }^{9}$ S. Swaminarayan and R. LeSar, presented at the Combined Workshops on Fracture, Friction, and Deformation, held at the Center for Nonlinear Studies, Los Alamos National Laboratory, Los Alamos, New Mexico, April 19-24, 1996.

${ }^{10}$ S. C. Crow, AIAA 8, 2172 (1970).

${ }^{11}$ L. Kubin, private communication.

${ }^{12}$ M. Li, Jianling Xu, Brian Smith, and R. L. B. Selinger, in preparation.

${ }^{13}$ Y. Mishin, M. Mehl, D. Farkas and D. Papaconstantopoulos, to appear, Phys Rev. B.

${ }^{14}$ For a study of a related two-dimensional system, see S. Romano, Phys. Rev. B 42, 8647 (1990).

${ }^{15}$ D. C. Chrzan and M. S. Daw, Phys. Rev. B 55, 798 (1997).

16 Z. Suo, Acta Met. 42, 3581 (1994). 

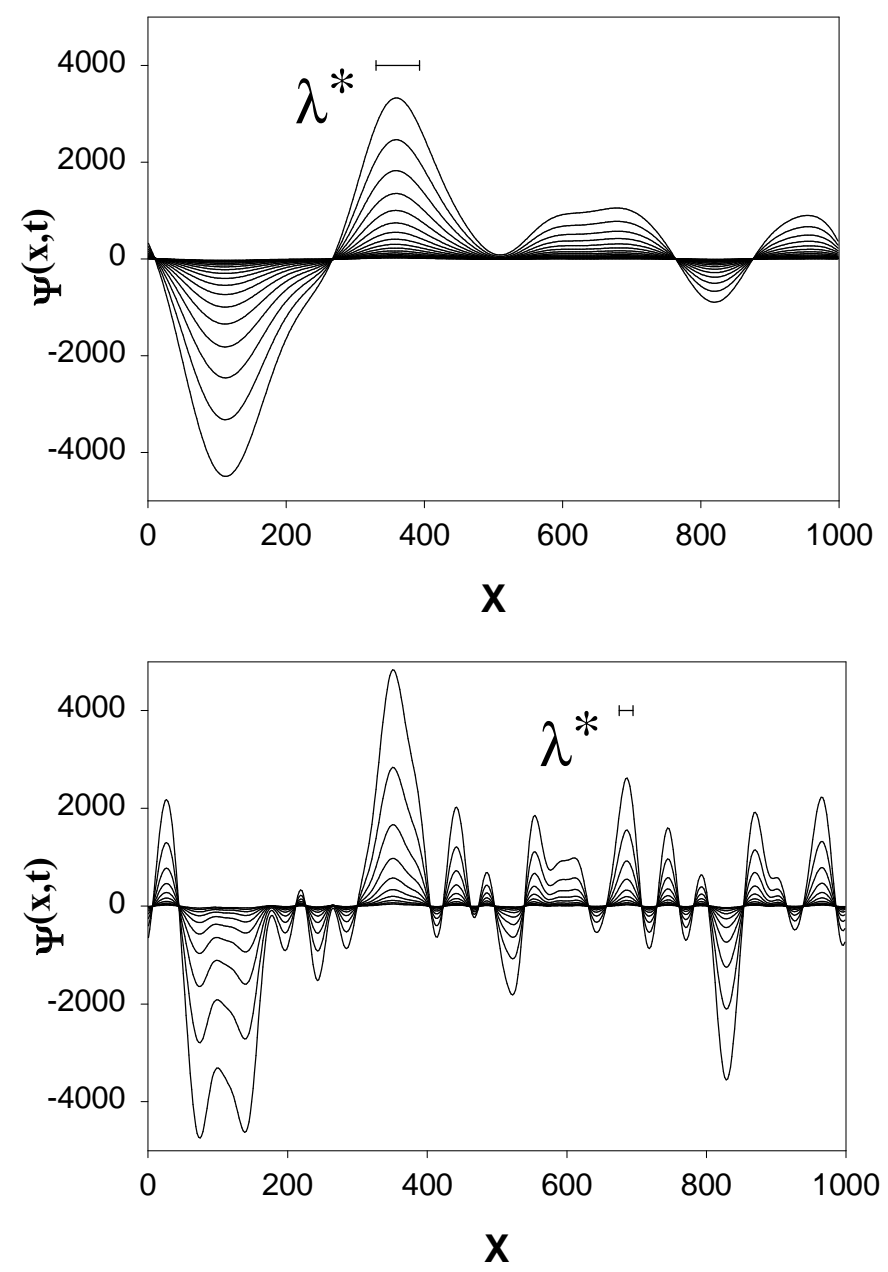

FIG. 1. Evolution of a line defect from numerical integration of Eqn. (10), $\gamma=0.1, c=1, m=1$, and $L=1000$. (a) With a weak force gradient $F_{1}=0.01, \lambda^{*}=63$. (b) With stronger force gradient $F_{1}=0.1, \lambda^{*}=20$. The pattern coarsens via tip combination. 


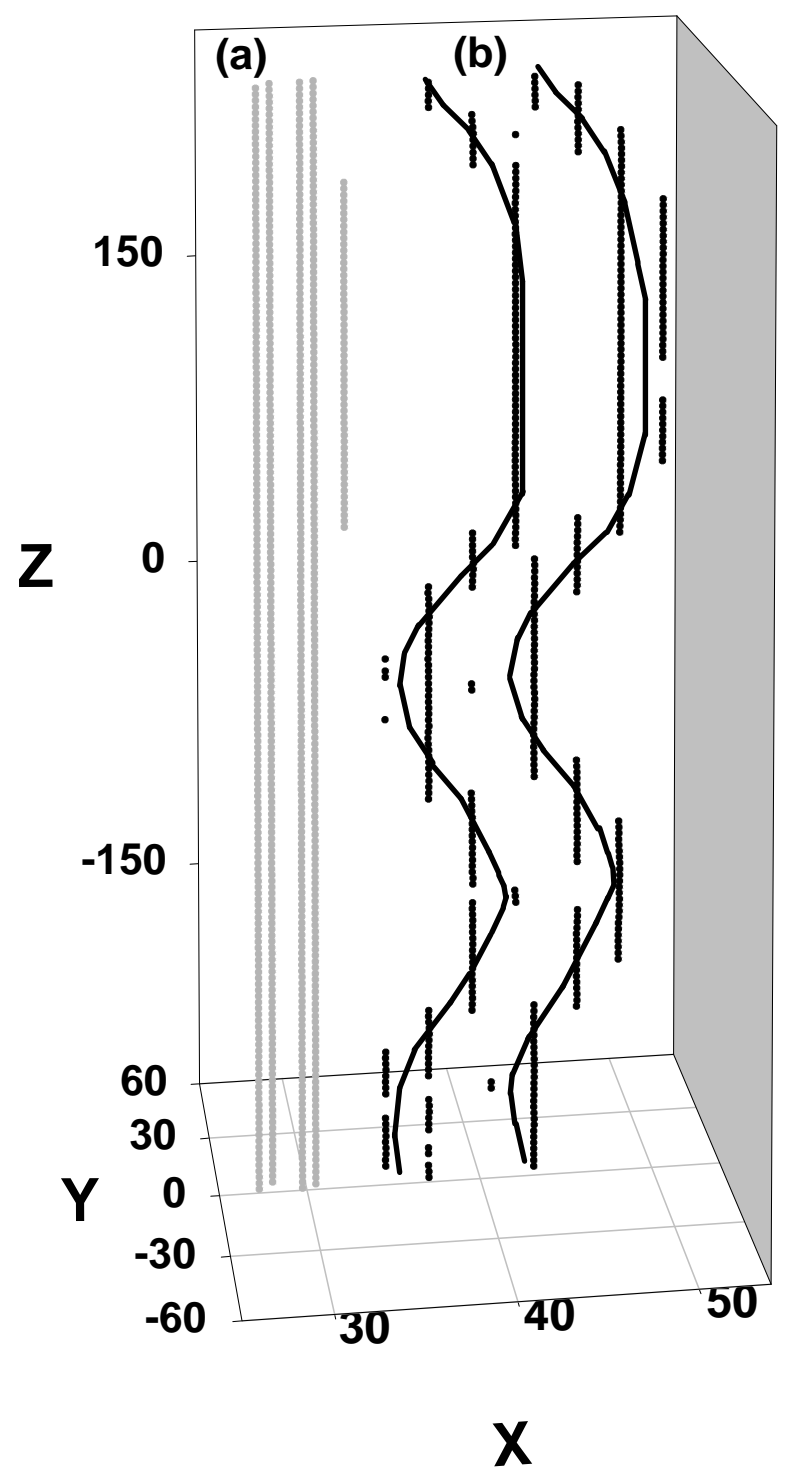

FIG. 2. Molecular dynamics simulation of screw dislocation motion in Al. (a) Initial configuration. (b) Later configuration; dislocation has split into partials which "finger" toward the free surface. Curves are drawn to guide the eye. 


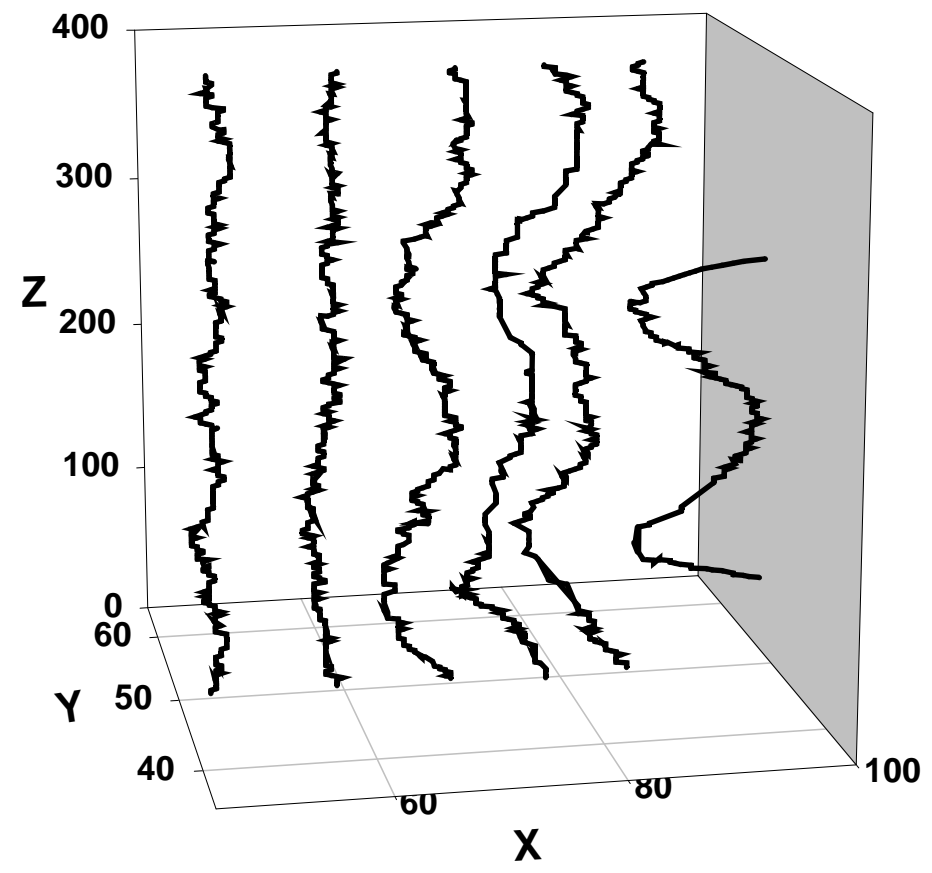

Fig. 3

FIG. 3. Time evolution of vortex motion in a 3-d XY "rotator" model simulation. As the vortex line approaches the free surface the first and then second vibrational modes become unstable. 